\title{
Novel large deployable antenna backing structure concepts for foldable reflectors
}

\author{
V. Fraux $\cdot$ M. Lawton $\cdot$ J. R. Reveles • \\ Z. You
}

Received: 13 February 2013/Revised: 11 July 2013/Accepted: 12 July 2013

(C) CEAS 2013

\begin{abstract}
This paper describes a number of large deployable antenna (LDA) reflector structure concepts developed at EnerSys-ABSL. Furthermore, EnerSys-ABSL has confirmed the desire to build a breadboard demonstrator of a backing deployable structure for a foldable reflector in the diameter range of 4-9 $\mathrm{m}$. As part of this project EnerSys-ABSL has explored five novel deployable structure concepts. This paper presents the top level definition of these concepts together with the requirements considered in the design and selection of the preferred candidate. These new concepts are described and then compared through a trade-off analysis to identify the most suitable concept that EnerSys-ABSL would like to consider for the breadboard demonstrator. Finally, the kinematics of the chosen concept is described in more detail and future steps in the development process are highlighted.
\end{abstract}

V. Fraux, M. Lawton, J. R. Reveles and Z. You contributed equally to this work.

This paper is based on a presentation at the ESA Workshop on Large Deployable Antennas, October 2-3, 2012, Noordwijk, The Netherlands.

\section{Fraux}

Cranfield University, Cranfield MK43 0AL, UK

e-mail: vincent_fraux@ @otmail.fr

M. Lawton $(\bowtie)$ J. R. Reveles

EnerSys-ABSL Space Products, Culham OX13 3ED, UK

e-mail: mike.lawton@absl.enersys.com

J. R. Reveles

e-mail: juan.reveles@absl.enersys.com

Z. You

Department of Engineering Science, Oxford University,

Oxford OX1 3PJ, UK

e-mail: zhong.you@eng.ox.ac.uk
Keywords Antenna - Deployable Structure . Ka-band · Reflector - Spacecraft .

Telecommunication

\section{Introduction}

A high-maturity European large deployable antenna (LDA) capability is seen as strategically important due to the EU's trailing position behind that of the US. ESA has introduced a new patented concept-patent 568-and wishes to encourage and support the development of an EU capability to service telecommunications, earth observation (EO) and science spacecraft. LDAs in the diameter range of 4-9 $\mathrm{m}$ are seen as strategically important [3] due to their wide applicability to broadband satellite telecommunication services.

The future market requirement for LDAs is expected to be driven by demands from commercial, military and scientific sectors. Commercial demand from fixed and broadcasting satellite services together with multi-media networks (requiring satellites as an integral part of network infrastructure) represent the greatest demand segment.

The customers for LDAs are ultimately the satellite operators. It is of little surprise then that customer-product requirements are aligned to their commercial goals and product capabilities rather than to the demonstration of technical achievement. Customer primary requirements for LDAs can be summarised as follows [3]:

- High reliability

- Low cost

- Mass efficient

- Maintain high-reflector surface integrity

- High-stowage efficiency 
- Side attachment capability

- Acceptable deployment time

For these reasons, EnerSys-ABSL is interested to address the EU's telecommunication needs and has proposed to undertake the development of a LDA Reflector Structure. In this project, EnerSys-ABSL is conducting an investigation into the application of carbon fibre reinforced silicone (CFRS) [3] technology for the reflector surface material.

The design of the deployable structure takes into account the requirements from the CFRS shell-membrane to be accommodated.

This paper first presents the top level engineering requirements considered by EnerSys-ABSL for the deployable antenna design. Five concepts developed for this project are then illustrated and compared using a trade-off matrix based on an assessment of the fulfilment of the engineering requirements for each concept comparatively. In the definition and assessment of the concepts presented in this paper, the design features and functionality offered by ESA patent 568 [1] and by the main competitor equivalent product, AstroMesh (the industry state-of-the-art LDA [3]), have been considered. Finally, the preferred concept EnerSysABSL intend to develop further is presented in more detail.

\section{Top-level engineering requirements}

The top-level engineering requirements for the deployable structure are derived from the customer requirements and can be grouped as follows:

- High reliability: First and foremost the structure should be able to deploy and stow without locking. Mobility one kinematic chains [5] (Sect. 5 of this paper) offer a good starting point for the definition of the candidate LDA architecture [3].

- High accuracy: The structure has to provide the required number of fixing points to ensure the reflector surface accuracy is achieved [3].

- High stowed volume and mass efficiency: These are requirements which facilitate an objective comparison between dissimilar LDA architectures. These two parameters are intrinsically linked to show how commercially attractive a given LDA architecture appears at a first glance [2, 3].

- Stability: This parameter will be fully quantified at a later stage. However, the candidate LDA must offer a mechanically and thermally stable platform for the reflector to ensure accurate pointing during in-orbit operations.

- Mounting configuration: Ideally, the structure should lend itself to be side-mounted to the spacecraft.

- Actuation principle and controllability: Although these parameters have not been fully explored, it is felt that a kinematically efficient concept will simplify the engineering implementation of the LDA concept later on.

- Scalability and modularity: Ultimately, to commercialise the selected LDA architecture, the preferred concept should be scalable (essential) to achieve a range of diameters from 4 to $9 \mathrm{~m}$ and modular (highly desirable) to increase the flexibility of the backing structure final configuration.

\section{Concepts}

Several mechanism concepts have been considered as the foundation for a closed-link kinematic chain capable of servicing a foldable membrane-shell reflector. As with any other complex dynamic system, LDA backing structures pose an interesting challenge in which conflicting requirements need careful attention and a compromise between them must be sought.

In the definition of the EnerSys-ABSL deployable antenna structure architecture, six basic engineering requirements (derived from the product requirements) have been considered paramount in the successful definition of a working LDA demonstrator concept.

EnerSys-ABSL have settled for two low-mobility concepts as the starting point for the definition of the final LDA breadboard since, generally, low-mobility architectures include attractive features such as self-synchronisation and a reduced number of actuation points. The basic architectures and its variants are described below:

- Double ring pantograph based concept

- Plain

- Simplified

- Sarrus mechanism based concept

- Pantograph prismatic: Stowed and deployed configurations are contained within a multi-faceted (at least six faces) prism

- Pantograph pyramidal: Stowed and deployed configurations are contained within a multi-faceted (at least six faces) pyramid

- Pure sarrus pyramidal: Stowed and deployed configurations are contained within a multi-faceted (at least six faces) pyramid

These concepts are described in the following paragraphs.

\subsection{Double ring pantograph}

The double ring pantograph concept is formed of an outer dodecagonal (for instance) pantograph ring and a concentric, smaller hexagonal inner ring. The two rings are 

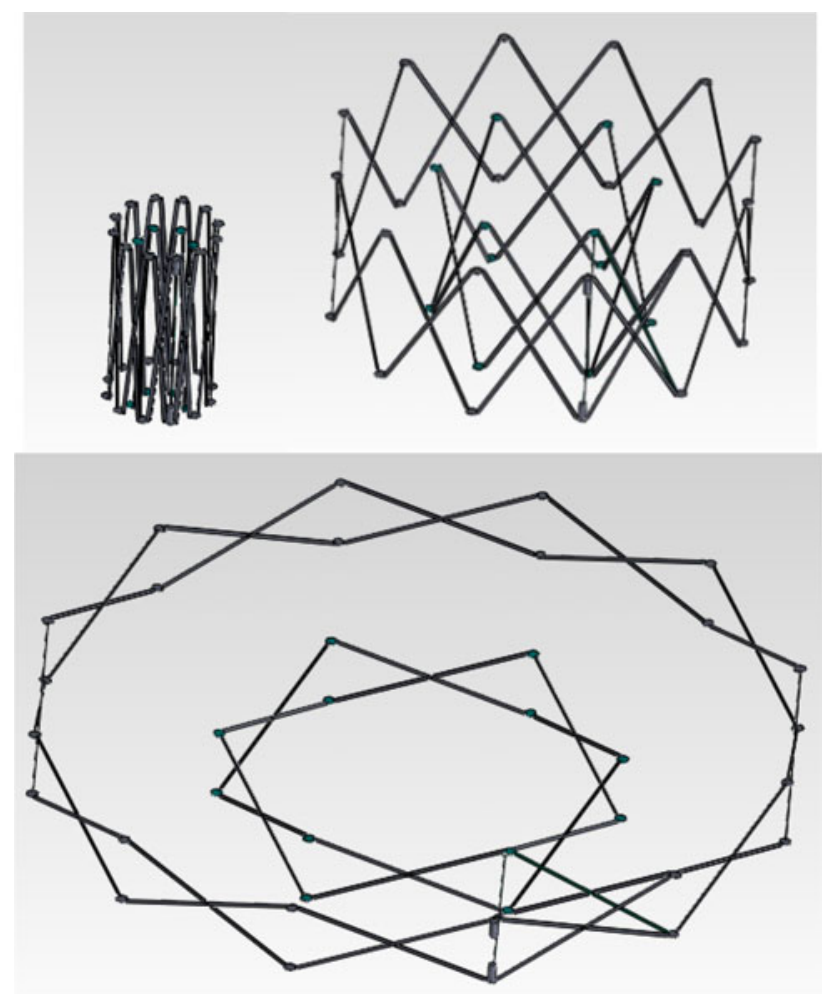

Fig. 1 Double ring pantograph-deployment (Note only 2 of the 12 scissor links are represented)

synchronised via radial scissor mechanisms linking the joints of the outer ring to those of the inner ring. The two rings are offsets from each other so that once deployed, the joint can support a curved surface. This kinematic chain displays mobility one, a kinematic constraint that would have to be relaxed during practical implementation of this concept (Fig. 1).

\subsection{Simplified double ring pantograph}

This concept is a lower mass variant of the double ring pantograph. The inner ring has been suppressed and the scissor mechanisms linking the two rings have been simplified.

The structure, and particularly the inner supports, is more flexible in terms of offset and positioning. The height in the stowed configuration is reduced as the protruding offset inner ring is removed. Cables can be used to stiffen the structure (Fig. 2).

\subsection{Sarrus-pantograph prismatic}

The Sarrus prismatic concept is a ring made of six-bar frames inspired by the Sarrus linkage design. The vertical bars (yellow) are linked via two V links in different planes ensuring them to be parallel and levelled. The optimal angle between the two planes in terms of structure stiffness

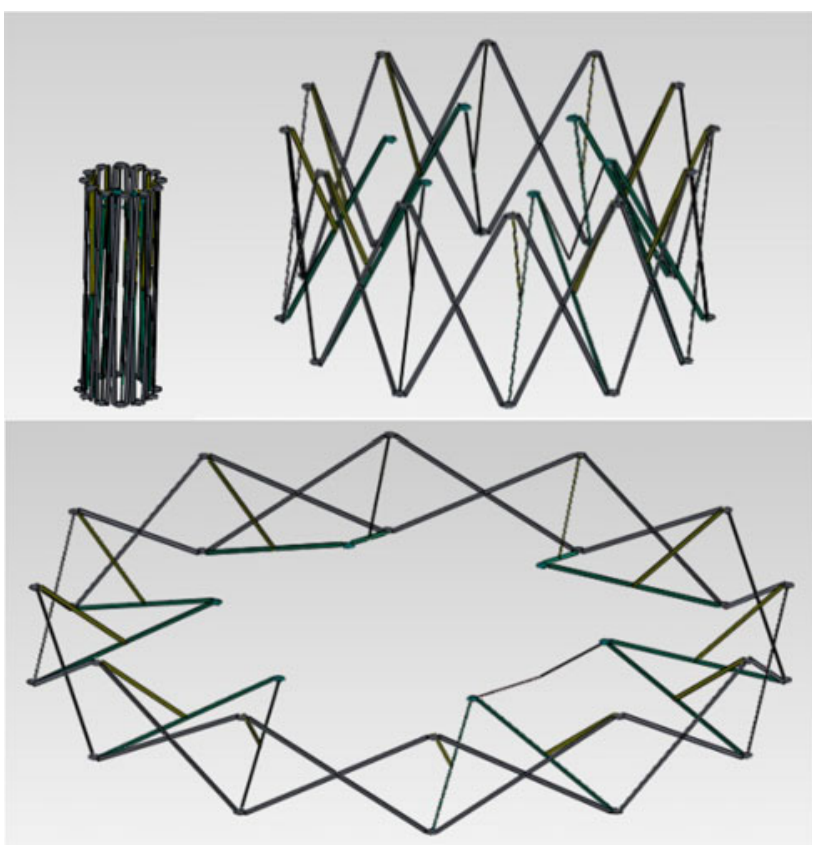

Fig. 2 Simplified double ring pantograph-deployment (Only 1 of the 6 re-enforcement cable is represented)

is $90^{\circ}$ but for stowed volume reduction, this angle can be reduced, possibly to $30^{\circ}$ or less.

The V link which is in the plane of the vertical bars (grey) is extended to become a scissor element forming a pantograph ring. The primary function of the pantograph ring is to synchronise the frames to obtain mobility 1 . Although it is not necessary for the kinematics of the mechanism, the pantograph permits the introduction of a prismatic joint along the vertical bar to stiffen the overall structure. Similarly to the ring pantograph concepts described above, relaxation of kinematic constraints during concept implementation will enhance deployability at the expense of controllability and actuation complexity.

The Sarrus-based concept does not provide any support inside the ring but it allows more volume for the surface reflector membrane when stowed (Fig. 3).

\subsection{Sarrus-pantograph pyramidal}

The Sarrus Pyramidal concept is an adaptation of the prismatic model. The vertical bars are now tilted by a chosen angle $\left(10^{\circ}\right.$ for instance) to form a hexagonal pyramid of twice this angle $\left(20^{\circ}\right)$. The pantograph elements are adjusted to maintain a constant angle between the neighbouring vertical bars and therefore maintain a constant pyramid angle. Although the conical version of the Sarrus concept is more complex, it is expected to be stiffer than the prismatic version. Crucially, it would also allow modularity, as long as the ring maintains a hexagonal shape (Fig. 4). 

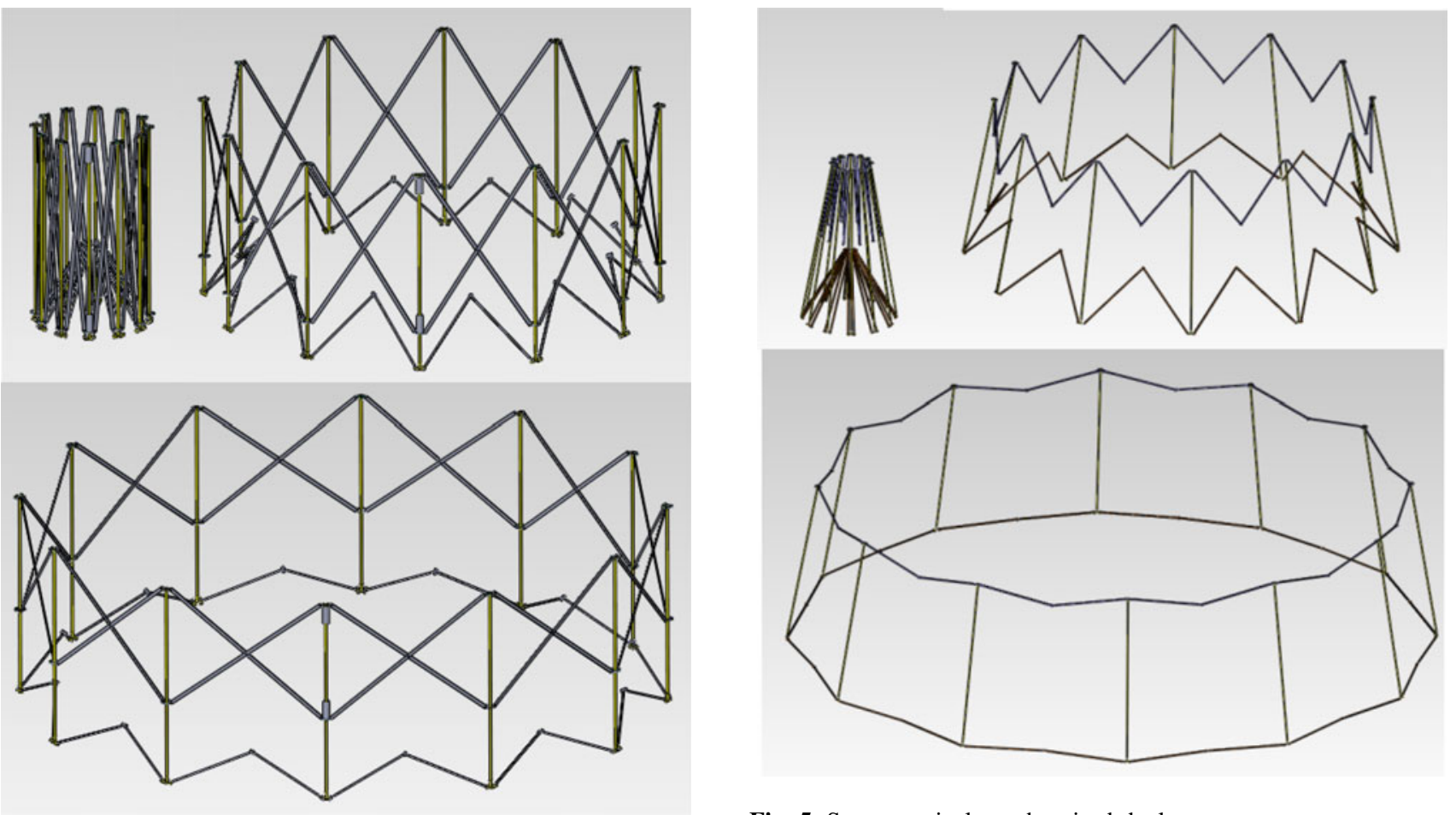

Fig. 3 Sarrus pantograph cylindrical-deployment

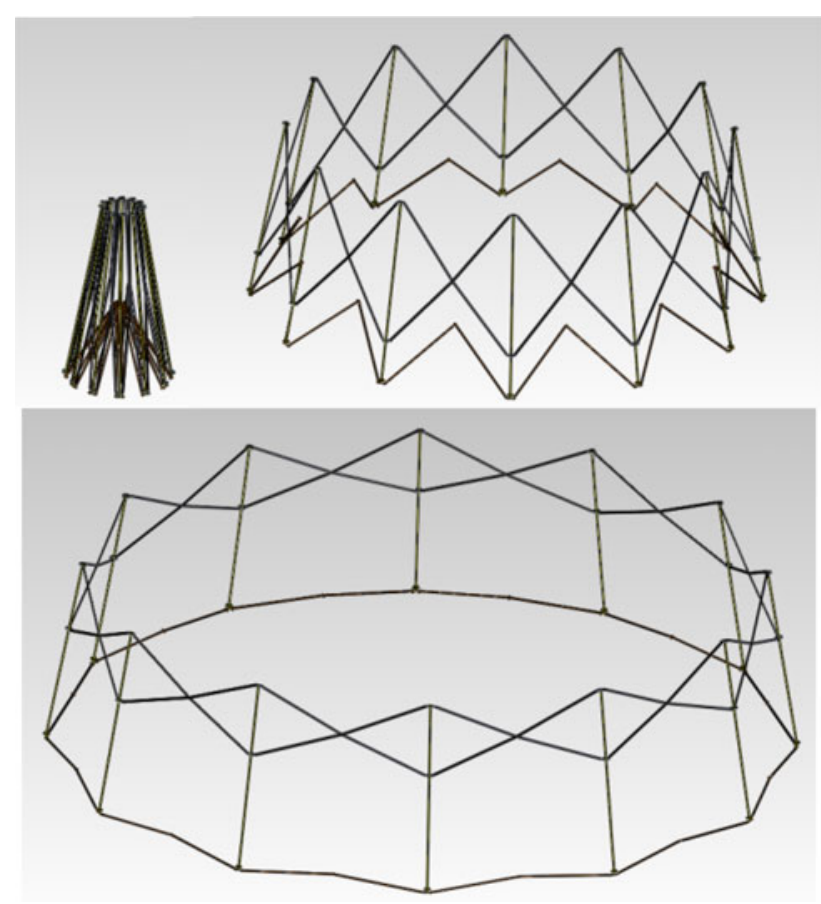

Fig. 5 Sarrus conical-synchronised deployment

previous concept in which the pantograph elements have been removed. This concept also allows modularity, provided the ring is in hexagonal shape.

Of the concepts described herein, the close kinematic chain obtained with the Pure Sarrus mechanism is the only concept that displays a mobility level greater than one, although each facet preserves its low mobility. In this concept, each Sarrus element is independent of the next which mitigates the risk of jamming. However, a lack of inherent self-synchronisation means an additional synchronisation system must be added together with an appropriate actuation system (Fig. 5).

For all the concepts mentioned in Sects. 3.1-3.5 in this paper, a combination of quantitative and qualitative preliminary analyses along with traditional CAD motion simulation packages have been conducted to validate the concept's design feasibility.

Consequently, the results obtained from these analyses and trade-off matrix played an important role in the down selection process of the best candidate. The selected candidate was then proposed for the definition and building of the LDA demonstrator. Further information on the concept selection process is provided in the following sections.

Fig. 4 Sarrus conical-deployment

\subsection{Pure sarrus pyramidal}

The Pure Sarrus pyramidal concept only uses the Sarrus mechanism at every facet and is an evolution of the

\section{Concept selection}

In order to down select which of the described concepts to take forward to the detailed analysis, design, build and test phases, they must be quantifiably assessed and compared. 
In this section, the five concepts are compared against each other. In the definition and assessment of the concepts presented in this paper, the design features and functionality offered by ESA patent 568 [1] and by the main competitor equivalent product, AstroMesh [3] have been considered.

\subsection{Concept selection process}

The concept selection process consists of a trade-off matrix in which parameters are defined to capture the engineering requirements that are relevant to this stage of the project.

Deployment reliability is judged to be of higher importance than the rest of the requirements, followed closely by mass, accuracy and stability. A scoring was assigned to each of the requirements; for example 1-5 factor according to their relevance in the parameter weight column in the trade-off matrix.

Sub-Parameter column offers detailed requirements, which are encompassed by the engineering requirement shown in the Parameter column. Each concept is scored according to their estimated degree of fulfilment of each sub-parameter.

The weighted average score of each category and for each concept is calculated using the score and weight attributed to each requirement.

Finally, the result of the trade-off matrix is given for each concept as a percentage score, the highest score highlighting the best concept. This is obtained by factoring in the weight of each Parameter.

\subsection{Trade-off result interpretation and recommendation}

The Trade-off matrix identifies all Sarrus mechanismsbased concepts as attractive candidates for the breadboard demonstrator. The highest scorer, the Pure Sarrus-based concept, preserves many of the characteristics and functionality displayed by ESA patent 568 [1, 4] including low mass, scalability, modularity and actuation complexity. It should be noted that the matrix table alone should not be viewed as the concluding deciding factor as to which concept to pursue, it should merely aid the decision process.

In light of exploratory work already undertaken on ESA patent 568, it is suggested that the pure Sarrus pyramidal concept be pursued in two stages:

- In the first stage of development, the self-synchronised pyramidal-Pantograph Sarrus mechanism (described in Sect. 3.4) will be considered.

- In the second phase, the Pure Sarrus pyramidal concept (described in Sect. 3.5) will be pursued. This can be viewed as an adaptation of the previous self- synchronised concept in which some elements of the pantograph will be removed to relax the kinematic constraints and increase the mobility of the chain. By removing the self-synchronicity of the original architecture, more complex controllability, actuation and synchronisation concepts will have to be considered.

\section{Sarrus pyramidal concept}

\subsection{Pure sarrus linkage}

The Sarrus mechanism is a special case of a spatial closed chain in which the Kutzbach criterion for mobility becomes zero (or negative in the case of the Sarrus mechanism with a pantograph element) [5]:

$m=6 n-5 j-6$

In Eq. 1, $m$ refers to the mechanism mobility; $n$ refers to the number of linkages and $j$ to the numbers of joints. This holds for a closed chain where only lower pair joints (e.g. revolute, prismatic, screw, cylindrical, spherical or planar joints) are involved and where each joint has one degree of freedom. Evidently, for a spatial closed chain to have mobility $1(m=1)$, seven links $(n=7)$ and seven joints $(j=7)$ are needed; any kinematic chain with fewer links and joints is to be either immobile or overconstrained. Immobile structures find no applicability in LDAs for obvious reasons. As for overconstrained mechanisms, they can be traced back to Pierre Frederic Sarrus who in 1853 reported a six-bar mechanism capable or rectilinear motion. In the current paper, we present a closed kinematic chain which following the accepted mobility criterion, can be described as a modified overconstrained six bar Sarrus linkage mechanism with six one-degree-offreedom (revolute) joints which displays mobility one.

This linkage arrangement allows the chosen angle $\alpha$ between the vertical bars (black bars in Fig. 6 above) to remain constant (Figs. 7 and 8). This characteristic is also

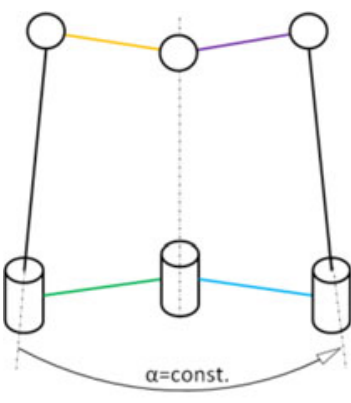

(a) Deployed

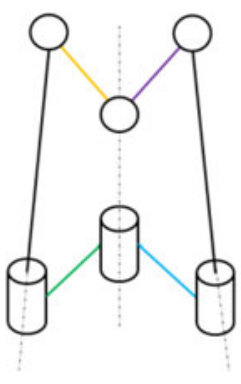

(b) Partially deploy

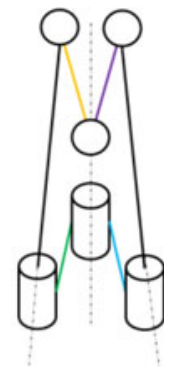

(c) Stowed
Fig. 6 Sarrus linkage schematic 

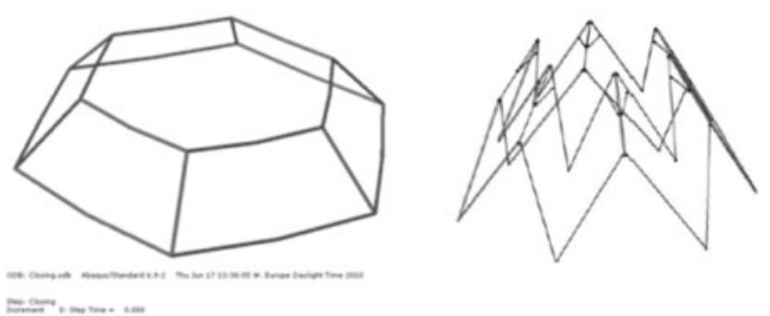

$=0+\cdots \cdots$

Fig. 7 ESA patent 568 [1]

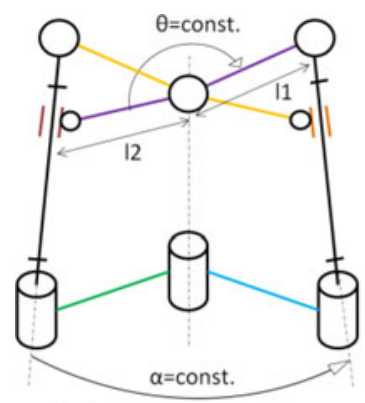

(a) Deployed

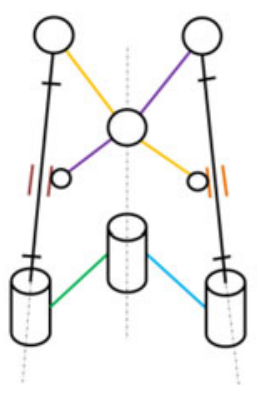

(b) Partially deploy

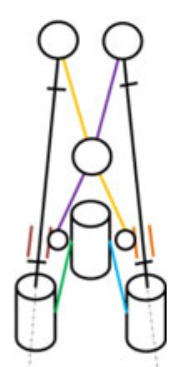

(c) Stowed
Fig. 8 Sarrus-pantograph linkage schematic

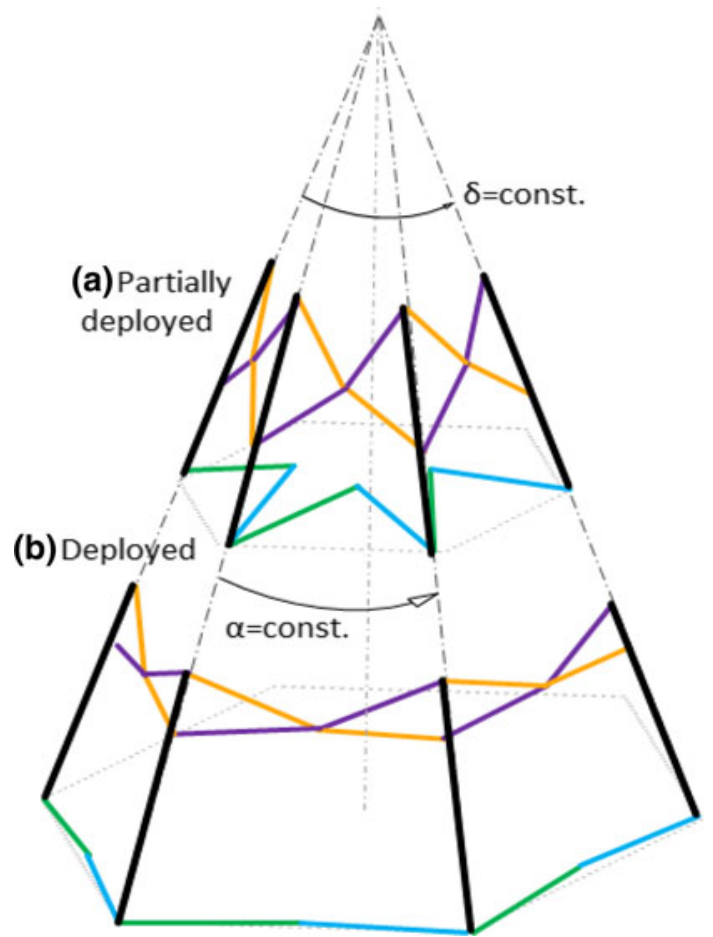

Fig. 9 Sarrus-pantograph hexagonal ring constant cone angle $\delta$ and $\alpha$ schematic

displayed by other deployable structure concepts such as the one developed and patented by ESA [4]. By carefully arranging the facet presented in Fig. 6 a hexagonal pyramid of constant angle can be obtained (Fig. 9).

\subsection{Sarrus-pantograph linkage}

- The pantograph is introduced into the Pure Sarrus mechanism as a simple means of synchronisation of all the elements of the close kinematic chain.

- For the pantograph sliding joints to follow the right path while deploying and maintain a constant angle $\alpha$ between the vertical bars, the following dimensioning rule has to be respected (Fig. 8) [5]:

$11=12$

$\theta=180--\alpha$

\subsection{Closed loop kinematic chain}

The vertical bars of the Pyramidal Sarrus-Pantograph Ring concept are tilted by a chosen angle $\left(10^{\circ}\right.$ for instance) forming a cone or pyramid of twice this angle $\left(\delta=20^{\circ}\right)$ as illustrated by (Fig. 9). The pantograph elements are adjusted to maintain a constant angle between the neighbouring vertical bars and therefore maintain a constant cone angle.

\section{Conclusion}

The LDA project at EnerSys-ABSL has led to the examination of five deployable structure concepts. A trade-off was made to support the choice of concept to be taken forward and developed into a functional breadboard demonstrator.

The Pure Sarrus Pyramidal concept scored highest out of the EnerSys-ABSL concepts. Nevertheless, it was decided that, as a logical progression in the development process, the Sarrus-Pantograph Pyramidal concept should be developed first and then adapted into a Pure Sarrus Pyramidal architecture, a concept that will pose more demanding synchronisation and actuation challenges.

\section{Further work}

The next steps of the EnerSys-ABSL deployable antenna project can be summarised as follows:

- Conduct detailed kinematic analysis of the sarrus pyramidal concept.

- Assessment of the different possible actuation architectures for both concepts.

- Conduct basic stress analysis of the structure to dimension structure joints and members.

- Conduct basic multibody dynamics to analyse the dynamic loads and structural performance.

- Conduct detailed design parameters trade-off studies to define the optimum configuration. 
- Produce detailed CAD models for the Sarrus-Pantograph Pyramidal concept which can easily be adapted into a Pure Sarrus Pyramidal concept.

- Production of a $4 \mathrm{~m}$ deployed diameter breadboard demonstrator.

- Conduct physical testing and demonstrate its kinematic capability.

Acknowledgments The Authors would like to thank the European Space Agency for their financial and technical support in this project.

\section{References}

1. World Intellectual Property Organisation, International Bureau. International application published under the patent cooperation
treaty-Low weight, compactly deployable support structure. International Publication Number WO2012/065619, 24 May 2012

2. Magliorelli, L., Scialino, L., Breunig, E., et al.: Reflector Technology Trade-off for LDA on Telecom and Earth Observation Applications, Proceedings of the 33rd ESA antenna workshop on challenges for space antenna systems, ESTEC Noordwijk, The Netherlands, pp. 18-21 October 2011

3. Mangenot, C., Saniago-Prowald, J., Van T'Klooster, K., et al.: Large Reflector Antenna Working Group-Final Report. s.l., ESTEC, 8 September 2010

4. Santiago Prowald, J., Such Taboada, M.: Innovative Deployable Reflector Design, Proceedings of the 33rd ESA antenna workshop on challenges for space antenna systems., ESTEC Noordwijk, The Netherlands, pp. 18-21 October 2011

5. You, Z., Chen, Y.: Planar double chain linkages. In: Motion Structure. Deployable Structural Assemblies of Mechanisms., Spon Press 2012 